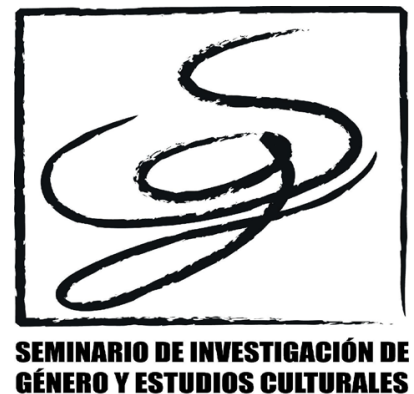

\title{
Las grafiteras, artistas y muralistas transgrediendo los roles de género dentro de la gráfica urbana de Aguascalientes
}

The graffiti women, women artists and muralists transgressing gender roles within the urban graphics of Aguascalientes

Sergio Raúl Recio Saucedo Universidad Autónoma de Ciudad Juárez sergio.recio@uacj.mx

Fecha de recepción: 01/07/2020 Fecha de evaluación: 20/09/2020 Fecha de aceptación: 14/11/2020

\begin{abstract}
:
The activities of graffiti, street art and urban muralism are characterized by being practices that have been linked mainly to the male figure due to the physical risks they pose to the participants. However, gradually women have been incorporated into the dynamics of graphic intervention of the spaces of the hydro-warm territory. Therefore, the article explains the gender stereotypes and the acts carried out by women graffiti artists, artists and muralists to transgress the cultural and historical ideas that limit and condemn their performance within the graphic-street movements of the city of Aguascalientes. Female graphic activity was approached from gender studies to elucidate the complexity of the cultural elements that socially define female and male identities within society. In addition, to analyze the dynamics that disturb the established orders destined to the transgression of the ideas that determine the duty of the female figure. This was possible through the application of in-depth interviews that provided information about their work in the movements. The results that were obtained were associated with the understanding of the acts that women use to transgress gender roles in the graffiti, street art and muralism movements. As well as the practices they develop to stay active. This made it possible to conclude that women present themselves in public spaces as a person capable and courageous of reproducing the street dynamics of movements in Aguascalientes.
\end{abstract}

Key-words: women, paint, graffiti, transgression, gender, roles, interventions

\section{Resumen:}

Las actividades del grafiti, street art y muralismo urbano se caracterizan por ser prácticas que han sido vinculadas principalmente con 
la figura masculina debido a los riesgos físicos que les suponen a los participantes. Sin embargo, paulatinamente la mujer se ha incorporado a las dinámicas de intervención gráfica de los espacios del territorio hidrocálido. Por lo que en el artículo se explican los estereotipos de género y los actos que realizan las mujeres grafiteras, artistas y muralistas para transgredir las ideas culturales e históricas que limitan y condenan su actuación dentro de los movimientos gráfico-callejeros de la ciudad de Aguascalientes. La actividad gráfica femenina se abordó desde los estudios de género para dilucidar la complejidad de los elementos culturales que definen socialmente a las identidades femeninas y masculinas dentro de la sociedad. Además, de analizar las dinámicas que perturban los órdenes establecidos destinadas a la transgresión de las ideas que determinan el deber ser de la figura femenina. Ello, fue posible a la aplicación de entrevistas a profundidad que proporcionaron información sobre su quehacer en los movimientos. Los resultados que se obtuvieron se asociaron con la comprensión de los actos que utilizan las mujeres para la transgresión de los roles de género en los movimientos del grafiti, street art y muralismo. Así como, las prácticas que elaboran para mantenerse activas. Esto, posibilitó que se concluyera que la mujer se presenta en los espacios públicos como una persona capaz y valiente de reproducir las dinámicas callejeras de los movimientos en Aguascalientes.

Palabras clave: mujeres, pintas, grafiti, transgresión, género, roles, intervenciones

\section{Introducción}

El grafiti, street art y muralismo urbano son actividades en las que se observa una mayor presencia de la figura masculina debido a las dinámicas legales o ilegales de la práctica. Por ejemplo, los integrantes se caracterizan por la transgresión de la infraestructura, la circulación nocturna por los espacios públicos y la exposición de la integridad física. Ello, ha generado que más hombres participen en la irrupción gráfica de los espacios. Sin embargo, en los últimos años se ha comenzado a visualizar la integración de las mujeres en la creación de piezas en las calles, pero en menor medida, lo cual se debe a los estereotipos de género que prevalecen en las sociedades actuales y que definen las formas de actuación de ambos géneros. De ahí que se haya determinado histórica y culturalmente que los espacios cerrados son las áreas destinadas para la acción de la mujer.

Por lo tanto, en el presente artículo se explican los estereotipos a los que se enfrentan las mujeres y las acciones que desarrollan las grafiteras, las artistas y las muralistas urbanas para transgredir los prejuicios de género que afectan su participación y su permanencia dentro de los movimientos gráfico-callejeros de Aguascalientes. Dicha idea, surge por creer que los juicios de valor limitan el involucramiento y el crecimiento de la mujer en el grafiti, street art y muralismo hidrocálido, lo 
cual se ve reflejado en una menor presencia tipográfica, gráfica y figurativa sobre los diversos elementos de la infraestructura de la urbe.

En este sentido, el artículo se estructura en tres secciones: 1. El género y los estereotipos. Aquí se explica qué es el género y cómo determina el deber ser de la sociedad. 2. Aguascalientes, una mirada social al territorio. En este subtema, se explora el contexto sociocultural de la sociedad hidrocálida. Asimismo, en los apartados 3 y 4 se aborda el ingreso de la mujer a los movimientos gráfico-callejeros, así como los procesos que posibilitaron su involucramiento. En el subtema 5 se explican los estereotipos y las formas de transgresión que ha realizado la figura femenina para participar en el grafiti, street art y muralismo urbano de Aguascalientes.

\section{El género y los estereotipos}

La participación de la figura femenina en las dinámicas del grafiti, street art y muralismo urbano ha adquirido mayor relevancia al observarse un incremento de mujeres que intervienen las calles de manera gráfica y tipográfica. Sin embargo, el número de participantes es aún reducido en comparación a los hombres que realizan pintas en los espacios. Esta disparidad, es analizada mediante los estudios de género para el establecimiento de una conexión que devele las implicaciones sociales que les suponen a las mujeres adentrarse en la creación de piezas callejeras. Para ello, es importante saber en qué se enfocan los estudios de género.

En este sentido, los estudios de género se caracterizan por analizar la complejidad de los elementos culturales que definen socialmente a las identidades femeninas y masculinas dentro de la sociedad. Además, abordan las dinámicas de organización social y los ámbitos de producción y de reproducción a los que han sido destinados tanto hombres como mujeres. Es decir, su "objeto de estudio son las relaciones socioculturales entre mujeres y hombres (hombres y hombres/mujeres y mujeres) y parten de la premisa de que el concepto mujeres (u hombres) es una construcción social, y no un hecho natural” (González, 2009: 682). De ahí que se centren en indagar las relaciones de poder y de desigualdad que producen los papeles sociales que se les han asignado históricamente a las mujeres y a los hombres.

La construcción histórica de lo masculino y femenino implica entender el término género como el "conjunto de ideas, representaciones, prácticas y prescripciones sociales que una cultura desarrolla desde la diferencia anatómica entre mujeres y hombres, para simbolizar y construir socialmente lo que es "propio" de los hombres (lo masculino) y "propio" de las mujeres (lo femenino)" (Lamas, 2000: 2). El deber ser determina las características de las personalidades que asumen tanto mujeres como hombres puesto que se les han atribuidos nociones para describir a ambos géneros como la delicadeza y la maternidad en el caso femenino; y, la fuerza y la agresividad para los masculino.

Asimismo, con el género se devela el vínculo sexo-cultura que se aleja de los procesos naturales en la construcción de las identidades 
femeninas y masculinas para centrarse en las dinámicas sociales e históricas, ya que son las creadoras de las pautas de conducta que determinan las formas de actuación de las personas. Es decir, la "cultura marca a los sexos con el género y el género marca la percepción de todo lo demás: lo social, lo político, lo religioso, lo cotidiano" (Lamas, 2000: 4). Además, es la que desarrolla relaciones de poder desiguales, crea la división del trabajo y establece dinámicas rituales. Entonces, se entiende que las mujeres y los hombres son productos culturales porque son configurados por las tradiciones y las costumbres de las sociedades.

Por lo tanto, el género construye la identidad femenina y masculina a partir de ciertas prescripciones sociales que aumentan las desigualdades entre ambos géneros al otorgar mayores privilegios a unos y someter a otros. Por ejemplo, históricamente la personalidad de las mujeres ha sido asociada con la maternidad, el hogar y el cuidado, lo que ha generado que se vean limitada a ciertas actividades dentro de las casas, al contrario, los hombres son los proveedores que salen en busca del sustento económico. Es decir, los roles de género regulan las relaciones sociales de los seres humanos en la sociedad debido a que la categoría de género "ha ejercido control sobre las personas desde instancias históricas que preceden nuestra era tomando el dictamen de la anatomía biológica como punto de partida para constituirnos en hombres o en mujeres" (Rodríguez y Toro, 2002: 64).

Sin embargo, en las últimas décadas se ha generado un proceso en el que se pretende disminuir las diferencias ideológicas para alcanzar la igualdad en derechos humanos mediante la transgresión de los roles de género. La mujer de forma paulatina ha tomado conciencia de las implicaciones sociales que poseen los estereotipos que predominan la feminidad y la masculinidad. Por lo que ha ingresado en una dinámica que busca perturbar los órdenes establecidos a través de la transgresión de las ideas que determinan el deber ser de la figura femenina. Es decir, "la transgresión significa cruzar los límites materiales, sociales y simbólicos vigentes hasta ahora, extender el espacio de actuar, romper el tabú, transgredir lo que el individuo es y lo que le pertenece" (Kozielecki citado por Mamzer, 2006: 120).

La mujer transgrede los roles de género mediante el cambio de actitud y de accionar, lo cual les implica alejarse de las ideas que determinan la identidad femenina como delicada, sumisa y hogareña para construir su personalidad bajo nociones que expresen fortaleza y libertad. Con ello, las mujeres se colocan en el ámbito productivo y público puesto que han comenzado a mostrar su capacidad intelectual y transgresora para apropiarse de las actividades que antes eran exclusivas para los hombres y convertirlas en entidades equitativas. Esto, se refleja en la incursión de la figura femenina en los movimientos del grafiti, street art y muralismo urbano debido a que han combinado la cotidianidad con la adrenalina para participar en la intervención gráfica de la infraestructura de Aguascalientes. 


\section{Aguascalientes, una mirada social al territorio}

El contexto geográfico de la ciudad de Aguascalientes en el que se ubican las grafiteras, artistas y muralistas se caracteriza por pertenecer al estado de Aguascalientes, el cual se encuentra situado al centro de la república mexicana. Este, se distingue por ser una de las entidades más pequeñas del país al contar con $5616 \mathrm{~km} 2$. Sin embargo, la ciudad de Aguascalientes es el municipio con mayor extensión territorial, ya que cuenta con $1178.85 \mathrm{~km} 2$. Además, la urbe posee una población de 797010 habitantes, según datos estadísticos del INEGI, lo que la convierte en la ciudad que alberga más personas de la entidad federativa.

Asimismo, la ciudad de Aguascalientes sobresale por las condiciones que posee en infraestructura urbana, ya que la urbe "aparece entre las mejores posicionadas en el índice de Infraestructura de las Ciudades Mexicanas, toda vez que como zona metropolitana figura en quinto lugar con un puntaje estimado en 71.2" (Mora, 2019: s/p). Esto, significa que el municipio provee a la ciudadanía de equipamiento urbano de calidad, el cual incide en el crecimiento positivo de la región y de la población. Ello, se refleja, por un lado, en el desarrollo del sector inmobiliario, de salud y de educación. Por otro lado, se observa en el acceso a las telecomunicaciones, a la movilidad urbana de los habitantes y de las empresas para agilizar los traslados y para mantener conectada a las personas.

En la misma línea, el municipio de Aguascalientes se caracteriza por contar con "tres millones 379 mil 642 metros cuadrados de áreas verdes, distribuidas en parques, jardines, plazas públicas, triángulos y camellones" (Redacción, 2015: s/p). Igualmente, la urbe se encuentra configurada por múltiples terrenos baldíos, los que en conjunto suman alrededor de dos mil hectáreas del territorio que se encuentran en condiciones de abandono, ya que son zonas que carecen de funcionamiento público o privado. Por lo que se convierten en "focos de infección y de inseguridad en perjuicio de los habitantes" (Hernández, 2017: s/p).

El aspecto cultural que caracteriza a la ciudad de Aguascalientes se asocia con la religiosidad que profesan la mayoría de los habitantes. Es decir, los ciudadanos se adscriben principalmente a la religión católica transfigurando a la sociedad hidrocálida en la tercera entidad federativa con mayor número de personas que se consideran católicos. Según Valdéz (2012), el porcentaje de la población católica en el estado de Aguascalientes es del "93.2\%, lo que representa a más de 1 millón de personas, profesa la religión católica" (Valdéz, 2012: s/p).

Además, el contexto social de la ciudad de Aguascalientes se distingue, en primer lugar, por la estabilidad económica de la sociedad hidrocálida, lo cual se debe a diferentes razones. Una de ellas, es el crecimiento económico, ya que es uno de los estados que "creció 3.1 por ciento respecto al trimestre inmediato anterior, y 8.6 por ciento en comparación con el cuatro trimestre de 2017" (Rodríguez, 2019: s/p). Otra, es la tasa de ocupación y empleo que se ha creado en los primeros siete meses de 2019. Es decir, se generaron "306,014 empleos; de los 
cuales, el 90\% corresponde a empleos permanentes" (Redacción, 2019: $\mathrm{s} / \mathrm{p})$.

También, se observa la estabilidad de Aguascalientes a partir de las dinámicas de seguridad social y urbana que imperan en la ciudad, lo cual se materializa en el descenso de la percepción de inseguridad en un periodo de tiempo establecido. Es decir, se redujo la sensación de inseguridad del " $60.0 \%$ al $48.1 \%$ de junio de 2018 a junio de 2019" (Romo, 2019: s/p). Esto, significa que la mayoría de la sociedad hidrocálida no posee temor por el desarrollo de relaciones sociales en los espacios públicos como las calles, los parques, las plazas o semi públicos, como los museos, las universidades, los centros comerciales.

A pesar, de la percepción de seguridad en Aguascalientes se desarrollan diferentes problemáticas de tipo social asociadas, en primer lugar, con los suicidios. Este problema, ha convertido a la urbe en la primera entidad federativa con mayor número de personas que se suicidan, ya que "se tiene una tasa del 9.6 por ciento por cada 100 mil habitantes desde el año 2016" (Ramírez, 2019: s/p). En segundo lugar, se observa la violencia hacia la figura femenina, ya que el "60\% de las mujeres han sido víctimas de violencia de género" (González, 2019: s/p) lo que se concretiza en actos violentos vinculados con el abuso físico, psicológico, verbal, así como, con desapariciones y asesinatos de chicas.

\section{La conformación de la escena gráfica-femenina de Aguascalientes}

La ciudad de Aguascalientes se distingue por la estabilidad social que predomina en los procesos económicos, socioculturales y religiosos de la región. Ello, ha generado una percepción de bienestar y de seguridad entre la ciudadanía, ya que cuenta con fuentes de empleo, infraestructura, áreas verdes y certidumbre en la sociedad. Sin embargo, es posible la visualización de dinámicas violentas asociadas tanto con los suicidios como con la violencia de género. El contexto social invita a plantear una pregunta que se vincula con saber ¿cómo es la participación, la actuación, las intervenciones y los intereses de las mujeres dentro de los movimientos del grafiti, street art y muralismo urbano de Aguascalientes?

Para dar respuesta a la interrogante primero se señalan características generales del involucramiento de la figura femenina en el país. La presencia de la mujer en los movimientos del grafiti, street art y muralismo urbano a nivel nacional ha ido adquiriendo relevancia en los últimos años debido a la constancia de sus intervenciones sobre la infraestructura de las ciudades. Se observa un involucramiento similar al de los hombres, pero con expresiones o mensajes que "remiten a la condición femenina, estableciendo alguna referencia discursiva a algún elemento del discurso femenino o perteneciente a él" (García, 2018: 126)

El quehacer gráfico de las mujeres en México se ve reflejado con distintas personalidades quienes se han centrado en la realización de paste up. Por ejemplo, se encuentra Maldita Carmen, Paste up Morras, An Art Work que intervienen las calles de la Ciudad de México con el propósito de compartir mensajes de carácter feminista. Asimismo, en San 
Luis Potosí sobresale el colectivo Gráfica X Morritas que elabora ilustraciones sobre papel, los cuales pega en la infraestructura de la urbe. Además, está Janin Nuz quien se realiza murales de tipo contestatario y comercial. Asimismo, en la ciudad fronteriza de Juárez participan de manera colectiva Lovely, Mala, Nema y Poli con la agrupación Perras Bravas. Ellas se han apropiado de las calles bajo la dinámica del street art con la finalidad de emitir mensajes en contra de la violencia de género.

Bajo este contexto, la participación de la figura femenina en los movimientos del grafiti, street art y muralismo urbano de Aguascalientes se caracteriza, en primer lugar, por configurarse como una comunidad reducida de mujeres jóvenes que poseen una edad que va de los 19 a 40 años. En ella, sobresalen los nombres de Venuska, Libre, Crick, Fatha, Domic, Misik, Sinika, Sandy de Santos, Vanessa Tochin, Fersuigin, Iztheni, Paola Palacios, Ely Ely, Sandra Sánchez y Bety Vargas. Las participantes se distinguen por ser originarias en su mayoría de la ciudad de Aguascalientes y en menor medida de la Ciudad de México. Además, son estudiantes universitarias y profesionistas de los programas de arte y diseño gráfico principalmente. Así como, por poseer carreras truncas en las licenciaturas de trabajo social y por tener estudios de nivel medio superior. Asimismo, son trabajadoras en dependencias de gobierno, en estudios de tatuaje, en spas y en áreas de rotulismo.

En segundo lugar, la escena femenina se distingue por encontrarse en la etapa de iniciación, lo cual se fundamenta en dos nociones; una, se refiere a que aproximadamente son 15 mujeres que intervienen gráficamente los espacios públicos, lo que genera poco involucramiento e interés del colectivo femenino en la pinta callejera. Otra, se asocia con la corta trayectoria de las participantes en la elaboración de propuestas tipográficas y gráficas sobre la infraestructura. Por ejemplo, Venuska, Domic, Fatha, Crick, Misik, Sinika y Libre son grafiteras que comenzaron a escribir su nombre en las paredes desde hace nueve, siete y cinco años, lo cual las convierte en las jóvenes que poseen mayor experiencia. En la misma línea, se encuentra Ely Ely y Bety Vargas, quienes trascendieron la urbe para laborar en la Ciudad de México, Nápoles y en Aguascalientes. Pero, las demás jóvenes, tienen máximo dos años de adentrarse en la creación de murales, sticker o paste up.

En tercer lugar, la escena femenina de Aguascalientes se caracteriza por la poca o nula vinculación social que existe entre las integrantes de los movimientos. Es decir, las jóvenes saben de la existencia de una o dos mujeres que se ha involucrado en la gráfica callejera, pero, no mantienen comunicación con ellas. Esto, transfigura al colectivo femenino en un grupo donde prima la individualidad, los intereses personales, la inexistente cooperación y la cercanía con hombres. Ello, ha generado una escasa visualización en la sociedad de la participación de las mujeres como hacedoras de grafiti, de paste up, de sticker, de esténcil o de murales.

En cuarto lugar, la escena femenina se distingue por la integración de las mujeres a los movimientos mediante diferentes expresiones gráficas. Por ejemplo, en el grafiti se integraron Venuska, Domic, Fatha, 
Crick, Misik, Sinika y Libre; en el muralismo, se incorporaron Ely Ely, Fer Suigin, Pao Palacios, Sandra Sánchez, Iztheni y Bety Vargas. Además, Sandy de Santos y Vanessa Tochin ingresaron al street art mediante la realización de wheatpaste y de sticker. Su integración, se debió a los intereses personales que tienen por la búsqueda de reconocimiento, por la expresividad artística, así como, por el contexto social de la ciudad de Aguascalientes. Ello, ha posibilitado tanto la intervención lúdica de los espacios, como la elaboración de proyectos asociados con el medio ambiente, el suicidio, la utilización de las bicicletas y el rescate del tejido social.

\section{Ingreso de las mujeres a los movimientos gráfico-callejeros}

El involucramiento de la figura femenina en los movimientos del grafiti, street art y muralismo urbano de Aguascalientes se encuentra ligado directamente con la presencia masculina. Esto, se debe, por un lado, a que la escena callejera está conformada principalmente por hombres quienes controlan la dinámica de intervención de los espacios públicos, ya que legitiman o deslegitiman la participación de las personas. Es decir, se presenta una relación de poder que produce "una estructura de dominación, exclusión y marginación sobre lo que no está aceptado como norma en la sociedad" (De la Villa, 2013: 13). Por otro lado, los hombres adquirieron las funciones de instructor, de guía, de protector y de juez al interior de la comunidad gráfica, lo que generó forzosamente que el ingreso de las mujeres en la realización de pintas estuviera vinculado con la figura masculina.

La presencia masculina en los procesos de introducción de las mujeres se observó en la mayoría de los casos de las participantes, ya que fueron vinculadas u orientadas por un hombre sobre las dinámicas de intervención gráficas de las calles. Por ejemplo, Sandy de Santos y Vanessa Tochin estuvieron acompañadas por la figura de un hombre mientras se integraron al street art debido a que ellos se convirtieron tanto en referentes inmediatos de la práctica callejera como en amistades y en colaboradores durante las primeras experiencias en la apropiación gráfica de la infraestructura de Aguascalientes. Esto, se materializó en la explicación de la forma de actuación, del acompañamiento diurno o nocturno al momento de la colocación de paste up y de stickers. Así como, con la invitación a eventos de intercambio de piezas gráficas y el desarrollo de exposiciones de arte.

Un compañero de mi salón hace stickers, entonces,
dije me gustaría intentarlo, hice el diseño, lo hice para
cumplir con una tarea, pero, me gustó. Entonces, me
acerqué a él y me empezó a dar consejos de cómo
hacerlo, qué hacer para evitar a la policía y todo ese
tipo de cosas. Pero, gracias a ese amigo he hecho
algunos contactos y cosas así ( $\mathrm{V}$. Tochin,
comunicación personal, 10 de octubre del 2019).

Asimismo, en el resto de las integrantes estuvo presente la figura masculina durante su incorporación a la comunidad gráfica mediante 
invitaciones, explicaciones y cooperaciones referentes a la creación de pintas en los espacios públicos y privados de la ciudad. De ahí que el ingreso femenino a los movimientos callejeros de Aguascalientes sea un esfuerzo individual por acceder a las dinámicas de intervención gráfica que se encuentra dominada por el hombre. Es un esfuerzo debido al rompimiento de la tradición histórico-cultural que coloca a la mujer en el ámbito privado, lo cual las ha llevado a que adquirieran "conciencia de la propia identidad y autonomía personal, reivindicando la competencia y la presencia de las mujeres en el mundo del arte" (Muñoz, 2013: 238). Así como, del grafiti, street art y muralismo urbano.

Por lo tanto, la incorporación femenina en los movimientos callejeros es una muestra de la determinación personal que poseen las mujeres para participar en las dinámicas públicas de intervención gráfica mediante la creación de personajes, de piezas, de proyectos y de discursos que son colocados en las calles. La participación, se ancla en la capacidad individual y social que tienen para demostrar a la sociedad la habilidad, la agilidad y la destreza en la realización de pintas tipográficas, gráficas y figurativas sobre el equipamiento urbano. El involucramiento femenino representa un cambio simbólico de la invisibilidad a la visibilidad de la mujer en las dinámicas del grafiti, street art y muralismo, ya que se empieza a observa la presencia de las grafiteras, las artistas y las muralistas en las estructuras físicas y del pensamiento de la ciudad de Aguascalientes.

Entonces, la incorporación de Venuska, Libre, Crick, Fatha, Domic, Sandy de Santos, Vanessa Tochin, Fersuigin, Iztheni, Paola Palacios, Ely Ely, Sandra Sánchez y Bety Vargas, se trata de un acto osado, estético y social que busca la paulatina construcción de la imagen de la mujer dentro de los movimientos callejeros. La imagen es construida con cada intervención gráfica que realizan en las calles puesto que son acciones transgresoras que dotan a la figura femenina de una identidad individual y colectiva. Además, de "un poder, una dignidad y una fuerza humana extraordinarios, aún en las situaciones más terribles de la experiencia femenina" (Muñoz, 2013: 254).

\section{Estereotipo y violencia una forma de delimitación social}

El ingreso de la mujer a los movimientos gráfico-callejeros se encuentra supeditado por los prejuicios sociales que surgen desde el ámbito familiar, social y religioso. Los juicios de valor están dirigidos a la condición de la mujer, ya que consideran que el grafiti, street art y muralismo son prácticas exclusivas para hombres. Por lo que el involucramiento de la mujer es cuestionado y subestimado al grado de percibirlo como algo negativo debido a la realización de actividades alejadas a la esfera privada. Esto, es resultado de "la lógica que señala al ámbito doméstico como condición sine qua non de lo femenino interpela particularmente a las jóvenes [...] y que designa que el "lugar de las mujeres" está en el espacio doméstico" (Hernández, 2018: 67). Por lo tanto, los estereotipos coartan la participación de la mujer al atarlas a los roles culturales que predominan en la sociedad de Aguascalientes. 
Por tal motivo, las grafiteras, artistas y muralistas se han enfrentado a diversos prejuicios sociales asociados, en primer lugar, con cuestiones históricas y culturales que han conducido a que los habitantes hayan "crecido con un mito fundacional que genera una identidad férrea: el considerarse gente buena" (Solano, 2018: 150). Esta idea, limita la personalidad femenina debido a que les supone ser figuras femeninas buenas centradas en la realización de actividades domésticas al interior de espacios privados; $y$, alejadas de dinámicas de carácter indisciplinado y del espacio público. Por lo tanto, ser mujer que hace grafiti, street art o muralismo es considerado como negativo porque no continúan con la reproducción del modelo femenino.

Creo que como seres humanos merecemos un trato
igualitario hablando de oficios, derechos o en este
caso del grafiti, no sé quién les dijo que debe ser
merecido por sólo hombres. También, sé que hay
hasta mujeres que ofenden a otras mujeres por
hacerlo, a mi me ha pasado así, hasta por hombres, sé
que no es fácil realizarlo en cuanto a criticas. Pero,
como te decía es más cuestión de cada quien, en el
cómo tomar ese tipo de comentarios y seguir
poniéndole color a este mundo que se torna gris sin
importar lo que diga la demás gente, simplemente soy
yo. (Crick, comunicación personal, 19 de octubre del
2019)

La idea de gente buena se vincula con la religiosidad puesto que los habitantes pertenecen principalmente al catolicismo, lo cual ha generado un adoctrinamiento social que se refleja en una educación que ha inculcado "jerarquías de valores como mecanismos de control, orientándolas hacia determinados comportamientos, hábitos, actitudes y sentimientos repetitivos, asumidos sin ningún tipo de cuestionamientos, aceptados como única y auténtica verdad" (González, 2010: 469). Este pensamiento incide directamente en los hombres, pero sobre todo en las mujeres debido a que coarta la capacidad intelectual, imaginativa y expresiva de la figura femenina para mantenerlas en los contextos tradicionales de la reproducción con la intención de alejarlas de las dinámicas gráfico-callejeras.

Estamos hablando de una sociedad muy machista, Aguascalientes es una de las, digo que influye en esto, no me acuerdo si es el segundo o el tercer lugar en todo el país de una sociedad en catolicismo y eso en Aguascalientes ha generado una doble moral. Entonces, esta doble moral hace que también sea machista y sea la mujer más juzgada y pues más tomando en cuenta si hace una actividad que para mucha gente pues es delictiva. Entonces, eso obviamente limita cualquier expresión (Iztheni, comunicación personal, 9 de octubre del 2019). 
En segundo lugar, los prejuicios se asocian con la sexualización de la mujer dentro de los movimientos gráfico-callejeros porque ellas establecen principalmente relaciones sociales con hombres, ya que las intervenciones tipográficas o figurativas giran en torno al acompañamiento masculino. Esto, ha generado diferentes ideas donde la figura masculina cosifica a las grafiteras, artistas y muralistas debido a que consideran que su integración se limita a un mero acto sexual convirtiéndolas en acompañantes de un grafitero o considerándolas como jóvenes libertinas.

\begin{abstract}
Siento yo que se debe a que muchas mujeres no se atreven a hacerlo a pesar de que les guste, no lo ejecutan por miedo a ser juzgadas o discriminadas, ya que vivimos en un mundo lleno de estereotipos y muchas de las veces la gente opina y hacen comentarios como: Una mujercita no debería andar de vaga pintando en las calles, qué van a decir de ti, acaso no te respetas o acaso no te da miedo. Entre otros tipos de comentarios similares (Venuska, comunicación personal, 18 de octubre del 2019).
\end{abstract}

En tercer lugar, las grafiteras, las artistas y las muralistas se enfrentan a un tipo de discriminación social por su condición femenina dentro de la comunidad gráfico-callejera. Ello, se observa en las dudas que surgen principalmente desde los hombres sobre la capacidad física e intelectual que poseen las mujeres para desarrollar pintas en los espacios públicos de la ciudad. Es decir, desconfían que el colectivo femenino pueda realizar actividades que supongan cierto grado de peligrosidad y de individualidad en las calles. Así como, de exposición pública, de la agilidad y de la destreza para moverse por los espacios. Además, de la utilización correcta de los materiales con los que se elaboran las propuestas gráficas.

Es complicado mantenerse porque es un movimiento que en su mayoría hay hombres y se presta a muchos comentarios machistas digámoslo así, ya que nunca falta el típico comentario de: "hay es mujer y por eso tiene fama" o "ya pinto con fulano de seguro ya se quiere colgar de su fama". Claro que si es difícil mantenerse en el movimiento (Venuska, comunicación personal, 18 de octubre del 2019).

En cuarto lugar, se suma la percepción que posee la sociedad sobre las prácticas del grafiti, street art y muralismo porque predominan concepciones que califican a los movimientos gráficos como actividades que ensucian y entorpecen el paisaje urbano de la ciudad de Aguascalientes. Estas ideas, se materializan, por un lado, reduciendo a la comunidad callejera a grupos vandálicos asociados con la cultura chola; por otro lado, minimizando las expresiones gráficas a marcas ilegibles que carecen de un sentido estético. Además, consideran a las pintas como problemas que afectan la imagen urbana, ya que contaminan visualmente la infraestructura debido a la proliferación de las piezas tipográficas, gráficas y figurativas. 
Yo siento que cualquier raya, cualquier pinta, cualquier tag lo encuentran como feo, lo encuentran como que hay que taparlo, como esto no pertenece, así como que no nos gusta, porque no se ve bonito. Entonces, cuando conciben el muralismo es de decoración, es bonito y es para una institución, es alegórico de lo que hable esa pequeña población que convive con ese mural. Entonces, si es más como de decoración y de visto bueno (S. de Santos, comunicación personal, 8 de octubre del 2019).

Aunado a los estereotipos de género, las integrantes se han enfrentado a diferentes inconvenientes que limitan su capacidad de acción dentro de los movimientos callejeros de Aguascalientes. Por ejemplo, reconocieron que se enfrentan a un aumento de la violencia social, ya que fueron víctimas de robo en diferentes áreas de la urbe como en las instalaciones de la universidad y la vía pública. Además, identificaron que existen zonas que son peligrosas e inseguras debido a que se ha desarrollado un proceso de descomposición social dentro de distintas colonias localizadas en la periferia.

Yo considero que Aguascalientes es seguro, la verdad si ha habido casos, pero comparado con otros estados, es muy tranquila, si puedes andar aquí a la una de la mañana y no pasa nada. Obviamente si andas en zonas peligrosas si puede ser. Pero, en general es muy tranquilo. Yo siento que no hay mucho problema en eso ( $P$. Palacios, comunicación personal, 11 de octubre del 2019).

Asimismo, las integrantes reconocieron que desafían constantemente la idea de encontrarse dentro de un espacio abierto, público, iluminado-oscuro y solitario, ya que pueden ser detenidas por las autoridades de seguridad pública durante la pinta de seudónimos o la colocación de sticker y paste up. Además, se enfrentan al miedo de convertirse en objetos de detenciones y de robos tanto de la policía como de la delincuencia, lo cual se debe tanto a la exposición de la integridad física como a la percepción de corrupción que generan las instituciones de seguridad en la ciudadanía.

El hecho de que sean más hombres que mujeres se
debe a que el trabajo es pesado físicamente, y
psicológicamente tienes que tener muchas
precauciones de todo tipo sobre todo viviendo en un
país que es poco seguro. Si pintas en la calle estás
expuesta a todo tipo de situaciones que por ejemplo el
hombre pasa por desapercibido (B. Vargas,
comunicación personal, 5 de noviembre del 2019).

También, las participantes reconocieron la existencia de dinámicas de acoso hacia la figura femenina tanto en los espacios públicos como en los espacios privados de Aguascalientes debido a que fueron víctimas de hostigamiento por parte de hombres. Ellas identificaron el acoso como un 
silbido, un piropo, un grito, una grosería, una mirada. Además, de tocamientos, de persecuciones y de asesinatos de mujeres. La percepción del acoso entre las grafiteras, artistas y muralistas aumenta dependiendo del tipo de movilidad urbana que emplean para trasladarse en la urbe. Por ejemplo, las integrantes que son peatonas, pasajeras o ciclistas han experimentado un mayor número prácticas de acoso, ya que las políticas públicas no las protegen ante tales situaciones.

Pues yo creo que en general es muy difícil que una
mujer pueda andar segura y ya sé que viene eso de
que tampoco los hombres porque nos asaltan y todo
esto. Pero, viéndolo por este lado de que a nosotras
nos pueden violar. Con mi círculo cercano, pues he
preguntado: ¿Oye a ti te ha pasado? Pues todas me
han dicho que si y todas han sufrido acoso, ya sea
desde el más mínimo como un chiflido hasta que te
rosan o cosas así. Entonces, a mí afortunadamente en
las calles no me ha pasado nada. Pues miradas
lascivas, que si me chiflan, que me hace sonidos, se
me quedan mirando, cosas así. Pero, por ejemplo no
me han tocado en la calle, pero si he vivido
experiencias fuertes de personas cercanas o de cosas
así. Entonces, en las noches caminando me siento
insegura, cuando salgo sola y traigo una falda me
siento insegura (V. Tochín, comunicación personal, 10
de octubre del 2019 ).

El acoso callejero se convirtió en una doble problemática social primero al afectar la integridad física del colectivo femenino; segundo, al normalizar las acciones negativas que nulifican los signos de hostigamiento sexual y justifican los actos como inocentes y carentes de maldad. Ello, despoja a los hombres de todo elemento de malicia para transfigurarlos en individuos ingenuos que no conocen sobre procesos que denigran y cosifican a la mujer, lo cual es resultado de los aprendizajes familiares e históricos donde se acepta las actitudes ofensivas de los hombres hacia las mujeres.

Pues yo creo que los hombres han sido, quieras o no, inconscientemente domesticados a ser de una cierta manera. Pero, yo creo que a veces son más nobles de lo que creemos, no todos, obviamente, no estoy justificando, ni diciendo que sean las personas más buenas del mundo. Pero, me pasó curiosamente hace tres días que iba en la bici y justamente un chavo que se paro así y me dijo ¡chula! y yo le conteste ¡chulo! Nos reímos los dos y yo seguí en la bici. Entonces, yo creo que a veces, obviamente, siempre es molesto que, por ejemplo hoy pasé y me hicieron jshttt! A eso me refiero, cómo que tanto se respeta. A veces pienso que no lo hacen tanto por maldad. Es como te digo, simplemente es ver como una flor, como que algo que aprecian y es como de ¡mira, que guapa! ¡bonita! pero, 
también tú sientes la intención ( $P$. Palacios, comunicación personal, 11 de octubre del 2019).

Asimismo, las grafiteras, las artistas y las muralistas se enfrentan dentro del movimiento a diferentes formas de violencia de género como la sexualización de las participantes al considerarlas objetos de compañía para los integrantes masculinos puesto que dudan de las capacidades intelectuales de las mujeres. Además, restan importancia a la valentía que poseen debido que los hombres pretenden transfigurarse en figuras protectoras durante las intervenciones callejeras.

\section{La transgresión de los roles de género dentro de los movimientos gráfico-callejeros}

Los estereotipos de género, las dinámicas de acoso y la inseguridad afectan el desenvolvimiento de las mujeres dentro de los movimientos gráficos de Aguascalientes puesto que han llegado a ser objeto de ataques en los espacios públicos. Sin embargo, las integrantes han permanecido en la escena callejera por voluntad propia, lo cual se debe a la firmeza de "adquisición de una mayor autonomía, de la capacidad de autodeterminación, de medios que permitan a todos/as gozar de una mayor elección en la vida" (Charlier y Caubergs, 2007: 6). Es decir, las participantes toman consciencia de sus capacidades intelectuales y físicas para saberse aptas para adentrarse en la creación de pintas en las calles.

Por lo tanto, se trata de un proceso de transgresión que busca romper con las dinámicas sociales y culturales que dominan las actividades de la figura femenina. Esto, tiene la intención de realizar cambios sustanciales en las estructuras heteronormativas de los marcos ideológicos de las prácticas del grafiti, street art y muralismo mediante el ingreso, permanencia y consolidación de las mujeres en los movimientos. Así pues, la transgresión consiste en la modificación y la eliminación de los roles de género para la libre ocupación de un lugar en los movimientos gráficos-callejeros posibilitando la construcción de la imagen femenina dentro de los espacios que tradicionalmente han sido dominados por hombres.

El proceso de transgresión se observa en diferentes acciones que desarrollan las mujeres para integrarse y para participar en los movimientos del grafiti, street art y del muralismo. El primer acto se asocia con la toma de decisiones sobre la incorporación a la comunidad gráficacallejera debido a la superación de las restricciones sociales que existen sobre la figura femenina. Aquí, las mujeres se proyectan como un ser humano libre y autónomo capaz de realizar cualquier actividad de índole pública. Por lo tanto, se adentran en una cierta dinámica inconsciente de empoderamiento al entrar, "en una visión de adquisición de poder, de control sobre su vida y la capacidad de hacer elecciones" (Charlier y Caubergs, 2007: 10). 


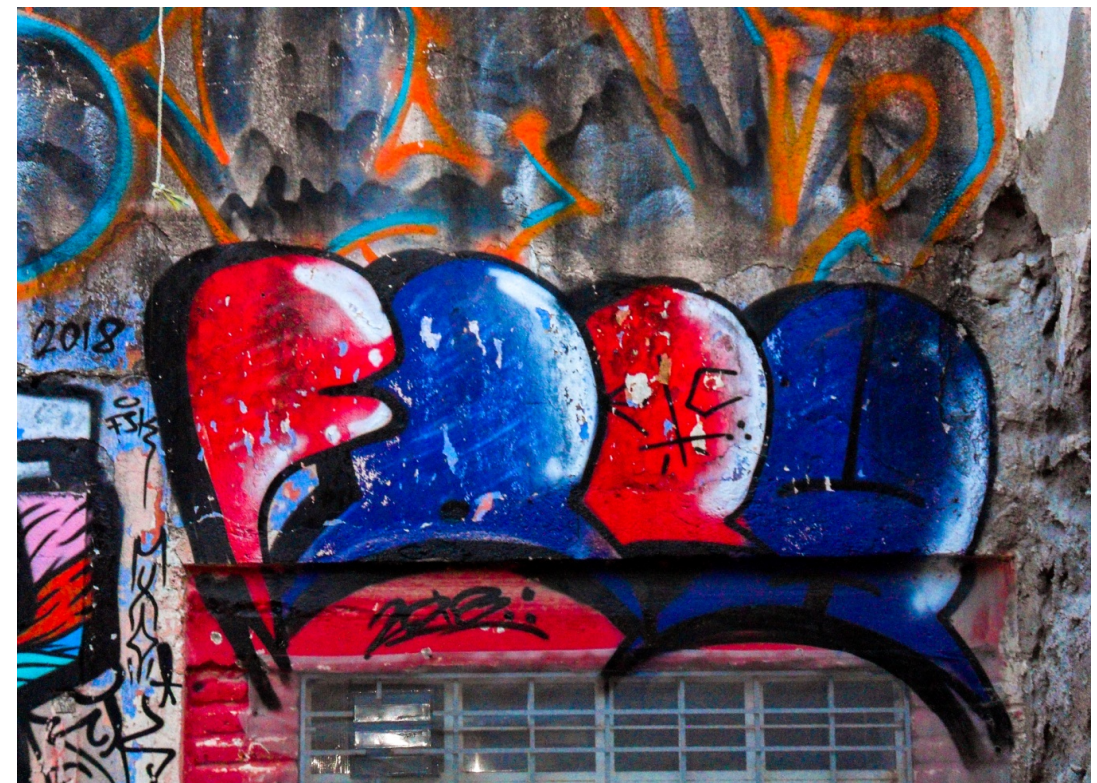

Fig. 1. Pieza de graffiti. Estilo Bubble letters. Elaborada por de Fatha. Calle Ramón López Velarde. Ciudad de Aguascalientes. Año: 2018. Fotografía del autor.

El segundo acto se refiere a la actuación de las mujeres en las calles de la ciudad de Aguascalientes, ya que transgreden el estereotipo del ámbito doméstico mediante la apropiación del espacio público para la realización de diversas actividades asociadas con los movimientos gráfico-callejeros. El accionar femenino en los espacios abiertos significa desprenderse de la percepción de la transitoriedad y de acompañantes al interior de las vías públicas para adentrarse en un proceso de exploración, de disfrute y de adquisición de poder que posibilita el libre proceder en el entorno urbano. Aquí, la actuación femenina expresa un tipo de revelación social donde la mujer termina entendiendo a las calles como escenarios urbanos dispuestos para la elaboración de prácticas; y, no sólo para relacionarse con cosas y objetos dentro de las casas. Por lo tanto, en las pintas tipográficas y figurativas se materializa el comienzo del posicionamiento de la mujer en el ámbito público. 


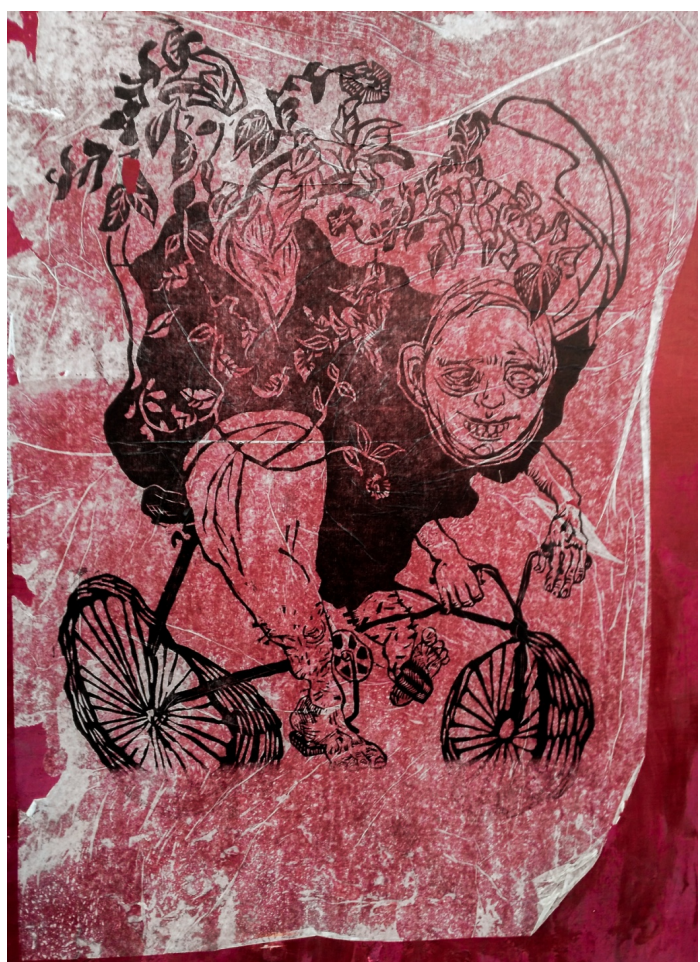

Fig. 2. Pieza de Paste up. Xilografía sobre papel de china. Propuesta elaborada por Sandy de Santos sobre el uso de la bicicleta. Av. Francisco I. Madero, zona centro de Aguascalientes. Año 2019. Fotografía del autor.

El tercer acto transgresivo se observa con la incursión de la mujer en la realización de pintas nocturnas sobre los espacios públicos debido a los peligros culturales que supone la oscuridad urbana. La noche es vinculada con el desarrollo de actividades ilícitas y criminales, por lo que las personas quedan expuestas y vulnerables ante identidades delincuenciales. La transgresión se visualiza mediante el desafío a la exposición nocturna para transfigurarse en figuras audaces que tienen la capacidad para moverse durante la noche. Es decir, las grafiteras, artistas y muralistas eluden distintos obstáculos en el ocaso como a las autoridades, a los delincuentes, además, de agresiones y de robos. Esto, busca quebrantar el orden público para aparecer gráfica y repentinamente en la infraestructura de la ciudad. 


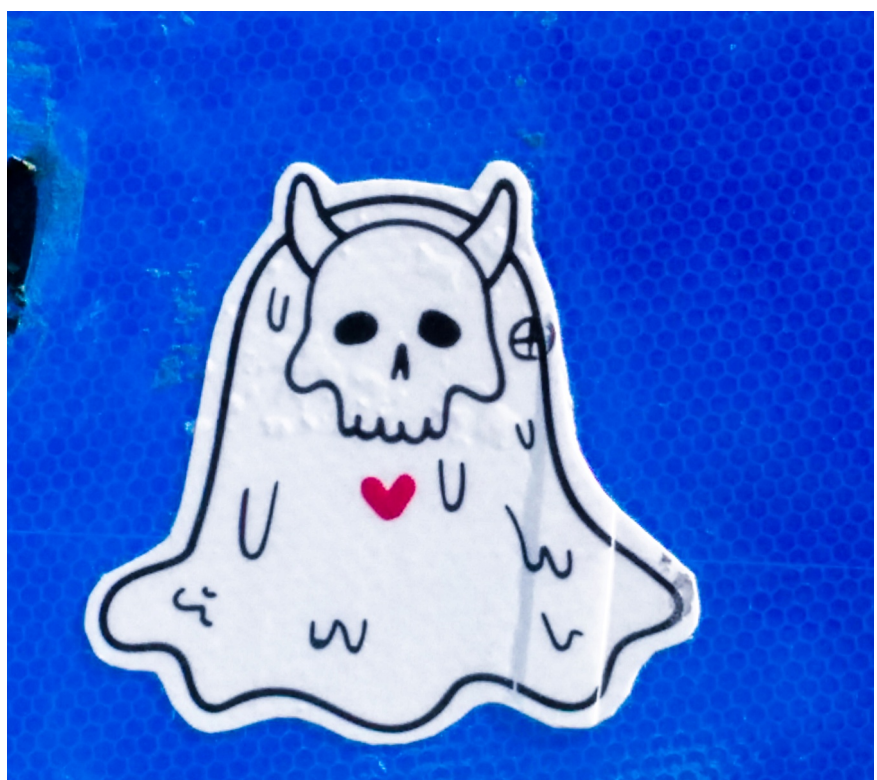

Fig. 3. Sticker de Vanesa Tochin impreso digitalmente sobre papel adhesivo. Propuesta que aborda la problemática de los suicidios. Afuera del Teatro Aguascalientes. Av. Aguascalientes Sur. Año: 2019. Fotografía del autor

El cuarto acto se asocia con el ingreso de la mujer a las dinámicas ilegales del grafiti, street art y muralismo, ya que culturalmente se considera que la figura femenina debe mantenerse dentro del ámbito legal y formal de la sociedad. Sin embargo, lo ilegal las reviste en personas capaces de actuar ocasionalmente al margen de la ley mediante la creación de piezas que quebrantan las reglas sociales para irrumpir la infraestructura con propuestas tipográficas y figurativas. Por lo que con el ingreso de ellas a los movimientos gráfico-callejeros se desarrolla un proceso que les posibilita construir identidades fuertes basadas en la valentía. Aquí, la transgresión se materializa en acciones gráficas que muestran el atrevimiento de las grafiteras, las artistas y de las muralistas en los espacios. Por ejemplo, con la pinta de tags, de bubble letters o con la colocación de wheatpastes y stickers en avenidas transitadas 0 edificios vigilados.

El quinto acto, se refiere a las técnicas y los mensajes que utilizan las grafiteras, las artistas y las muralistas para expresarse sobre la infraestructura. Por ejemplo, los contenidos se asocian a las dinámicas sociales que afectan a la sociedad como la problemática de los suicidios que es relevante para la población de Aguascalientes. Asimismo, hacen uso de materiales relacionados con pintura acrílica, papel, engrudo, pinceles y aerosoles para la creación de las piezas. 


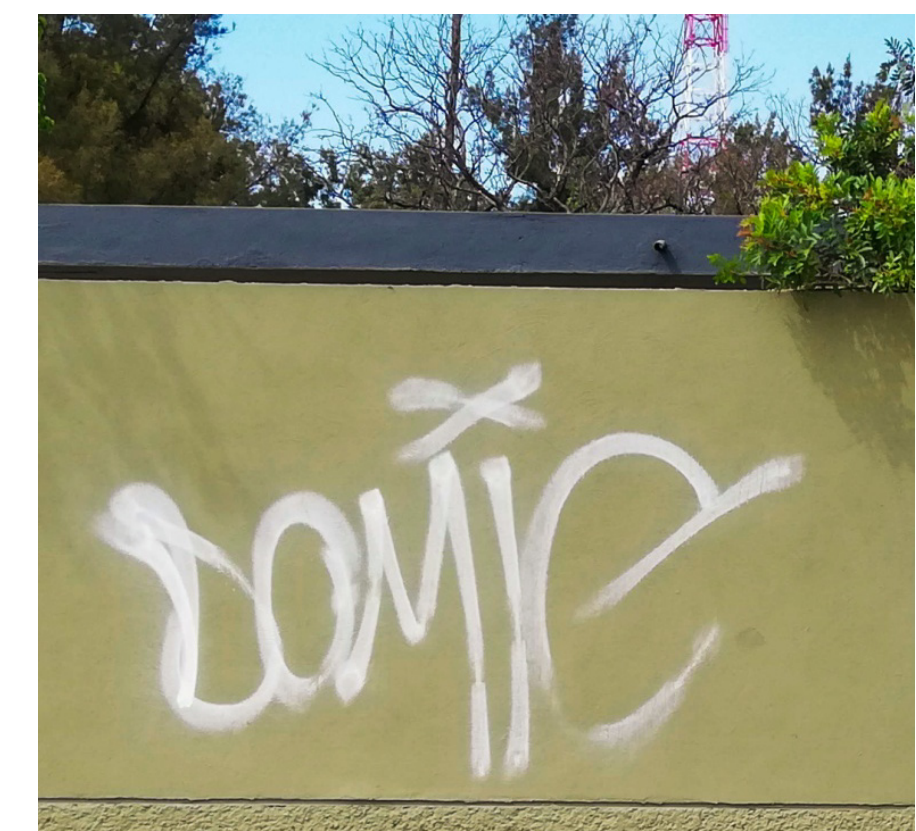

Fig. 4. Pieza de grafiti. Estilo tag. Propuesta elaborada por Domic. Av. Alameda. Ciudad de Aguascalientes. Año 2018. Fotografía del autor.

La transgresión de los roles de género produce que la presencia de la mujer en la calle signifique el comienzo de la reconquista de los espacios públicos, ya que se desarrolla un proceso que les permite acceder a los recursos espaciales de la ciudad de Aguascalientes. Aquí, la figura femenina se va apropiando de manera paulatina de los diferentes elementos que son parte del equipamiento urbano para participar activamente en su configuración mediante la creación de piezas tipográficas, gráficas y figurativas. Se genera un cierto protagonismo de la mujer en el ámbito público al adquirir conciencia sobre su condición en el entorno urbano. La reflexión les ha permitido que sean conscientes del papel que poseen en el grafiti, street art y muralismo "a partir de esta percepción sobre su contexto, puedan desarrollar su capacidad para cambiarlo, es decir, participen activamente en el proceso de transformación" (Orsini, 2012: 953). 


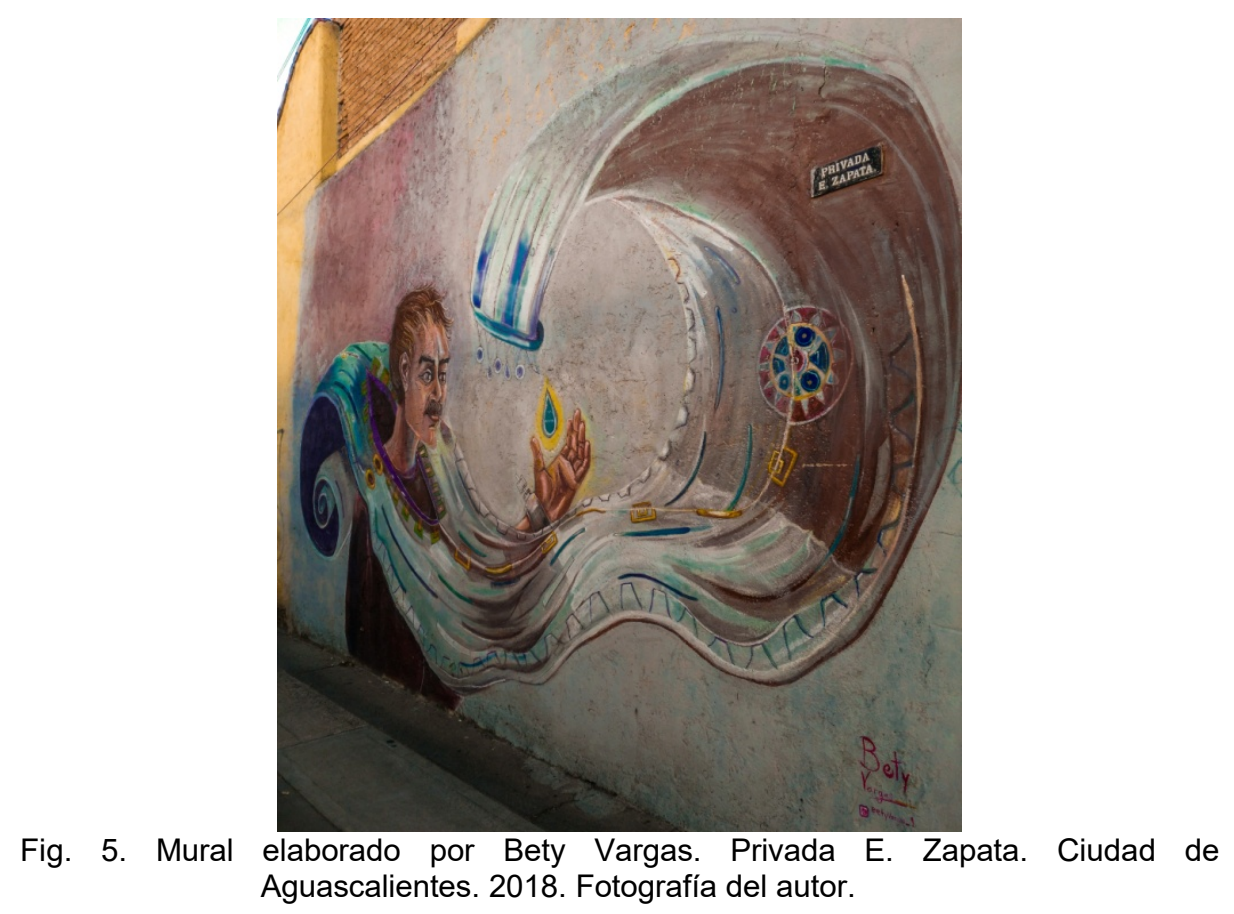

La concientización ha generado en la figura femenina la búsqueda de la permanencia dentro de las dinámicas de los movimientos gráficocallejeros para la construcción de la imagen de la mujer en la elaboración de piezas gráficas sobre la infraestructura. Entonces, la permanencia implica un esfuerzo individual y colectivo para la mostración de los trabajos, las piezas y las identidades de las mujeres al interior de la sociedad. Ello, produce diferentes procesos de aceptación y de consolidación que permiten la continuidad de las mujeres en la intervención de los espacios públicos.

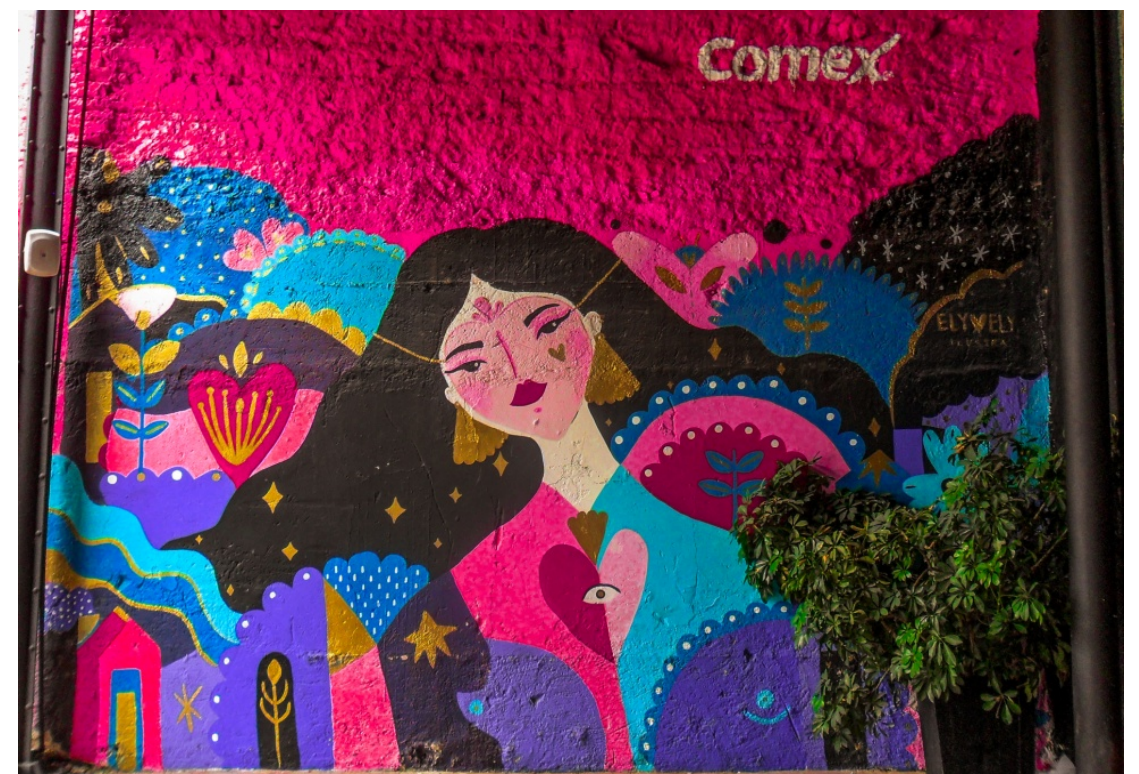

Fig. 6. Mural elaborado por Ely en el Quinto Barrio. Zona centro. Ciudad de Aguascalientes. 2018. Fotografía del autor. 
Así pues, la permanencia de la mujer en los movimientos ha sido un proceso complejo que les ha supuesto enfrentarse a las contradicciones sociales, económicas y culturales de Aguascalientes. Por ejemplo, tuvieron que eludir cuestiones asociadas con los costos de la pintura acrílica y en aerosol para continuar participando en los movimientos del grafiti, street art y muralismo. Asimismo, hicieron caso omiso a los comentarios sexistas, denigrantes y discriminatorios acerca de su participación en la creación de pintas. Al respecto, Venuska comenta que su permanencia en la escena femenina:

\begin{abstract}
Pues no ha sido fácil, ya que el costo del material se ha elevado muchísimo con el paso de los años, y, pues, no siempre se puede pintar, ya que existen prioridades a veces se puede y otras no. Lo importante es que seguimos en esto (Venuska, comunicación personal. 22 de octubre del 2019).
\end{abstract}

Asimismo, la permanencia de Sandy de Santos, de Vanessa Tochin y de Pao Palacios en la escena gráfico-callejera fue posible al apoyo que les han brindado familiares y amistades, ya que se convirtieron en personas fundamentales para la introducción, la continuidad y la realización de proyectos gráficos o la creación de murales. Es decir, los parientes y amigos de Sandy y Vanessa consideraron la participación de ellas como una actividad poca riesgosa en los espacios públicos al ser parte del proceso de aprendizaje de la formación universitaria. Además, reconocieron en Pao y Fer las capacidades artísticas que posee en relación al campo del arte, por lo que fueron invitadas para la elaboración y la colaboración de propuestas figurativas.

Por lo tanto, la permanencia de las mujeres en los movimiento gráfico-callejeros se debe al posicionamiento social al que se adscriben las grafiteras, las artistas y las muralistas puesto que son sus ideas las que han permitido la continuación en las actividades de intervención gráfica. Ello, determina la conducta y el actuar de las integrantes a nivel individual y colectivo reflejándose en la productividad gráfica y en las acciones que realizan en las calles. Por ejemplo, Iztheni y Vanessa Tochin se identifican con el feminismo y los estudios de género, lo que las ha llevado a preocuparse por las dinámicas sociales que afectan a la Ciudad de Aguascalientes.

Asimismo, Fersuigin se clasifica como liberal debido a la movilidad urbana y a los procesos de sociabilidad en la que participa cotidianamente que posibilitan interrelacionarse con personas que la vinculan con la creación de piezas muralísticas. Además, el resto de las integrantes se definen como neutrales ante los procesos sociales, ya que consideran que respetan las diferentes formas de pensamiento al interior de la sociedad, lo cual las ha llevado a involucrarse en el grafiti, street art y el muralismo para la búsqueda del reconocimiento de la identidad y de la calidad técnica que poseen. 


\section{Conclusiones}

La escena femenina del grafiti, street art y muralismo se estructura por una serie de elementos que indican que se encuentra en una etapa de iniciación debido a diferentes razones como la construcción social del género que condena a la mujer al ámbito doméstico y limita su presencia en los espacios públicos. Por lo que la participación de la mujer en el grafiti, street art y muralismo urbano de Aguascalientes implica pensarla en términos de transgresión debido al esfuerzo social que hacen para la creación de piezas gráficas en las calles. De ahí que la mujer se presente en los espacios públicos como una persona capaz y valiente de reproducir las dinámicas callejeras de los movimientos.

La transgresión del género ha llevado a las mujeres grafiteras, artistas y muralistas a que configuren una comunidad gráfica que se caracteriza por un número reducido de integrantes puesto que los movimientos callejeros se presentan a la ciudadanía como actividades negativas que no son propias del colectivo femenino. De ahí que la experiencia de las participantes sea corta en la intervención de los espacios al no encontrar suficiente apoyo de la familia como de las instituciones para adentrarse en la creación de piezas en las calles. Ello, ha generado una individualidad y a una actuación inconexa dentro de la práctica femenina, ya que las integrantes no se conocen, por lo que realizan esfuerzos particulares para sobresalir en el grafiti, street art o muralismo urbano.

\section{Referencias bibliográficas}

CHARLIER, Sohie \& Caubergs, Lisette. (2007). El proceso de empoderamiento de las mujeres. Guía metodológica. Francia: Femes y Developpement. Con sultado en: http://www.dhls.hegoa.ehu.eus/uploads/resources/4668/resource files/pr oceso empoderamiento mujeres CFD.pdf

DE LA VILLA, A. Rocio. (2013). Crítica de arte desde la perspectiva de género. Investigaciones Feministas , 4, 10-23. DOI: https://doi.org/10.5209/rev_INFE.2013.v4.41874

GARcía, Pardo Belén. "La calle como espacio artístico de mujeres: la Writer y la street artist.» Dossiers Feministes, $\mathrm{n}^{\circ} 23$ (2018): 125-141. DOI: http://dx.doi.org/10.6035/Dossiers.2018.23.8. Consultado en: http://www.e-revistes.uji.es/index.php/dossiers/article/view/3382/2875

GoNZÁLEZ JIMÉNEZ, Rosa. (2009). Estudios de género en educación. Una rápida mirada. Revista Mexicana de Investigación Educativa , 14 (42), 681-699. Consultado en: http://www.scielo.org.mx/scielo.php?script=sci arttext\&pid=S140566662009000300002

GonZÁLEZ, Marcela. (16 de febrero de 2019). 60\% de las mujeres han sido víctimas de violencia de género. Hidrocálido Digital. http://www.hidrocalidodigital.com/local/articulo.php?idnota=152951

GoNZÁLEZ, P. Teresa. (2010). Desigualdad, mujeres y religión. Sesgos de género en las representaciones culturales religiosas. Cuestiones de género: de la igualdad y la diferencia. (5), 467-505. Consultado en: 
http://revpubli.unileon.es/ojs/index.php/cuestionesdegenero/article/view/3 797/2673

HERNÁNDEZ G, Cristina. (10 de noviembre de 2017). Hay en Aguascalientes Cerca de 2 mil Hectáreas de Terrenos Baldíos. Página 24. https://pagina24.com.mx/2017/11/10/local/hay-en-aguascalientes-cercade-2-mil-hectareas-de-terrenos-baldios/

HeRnÁNDEZ, H. Luisa. (2018). Mujeres y grafiti en México, algunas reflexiones sobre género y juventud. Voces de jóvenes, 63-74. DOI: 10.1016/S01889478(16)30088-3

KOZIELECKI cita por Mamzer, Hanna. (2006). La identidad y sus transgresiones. La ventana (24), 118-149. Consultado en: http://www.scielo.org.mx/scielo.php?script=sci arttext\&pid=S140594362006000200118

LAMAS, Matha. (2000). Diferencias de sexo, género y diferencia sexual. Cuicuilco , 7 (18), 1-24. Consultado en: https://www.redalyc.org/pdf/351/35101807.pdf

MORA LEGASPI, Mario. (7 de enero de 2019). La ciudad con muy buena infraestructura. El sol de centro. https://www.elsoldelcentro.com.mx/local/la-ciudad-con-muy-buenainfraestructura-2891589.html

MuÑOz, L. Pilar. (2013). Arte feminista. Empoderamiento de las mujeres en el arte. El ejemplo de Paula Rego. Cuadernos Kóre. Revista de historia y pensamiento de género (8), 237-265. Consultado en: https:/lerevistas.uc3m.es/index.php/CK/article/view/2042/987

ORSINI, Marta. (2012). El concepto de empoderamiento en los estudios de género y en la prensa femenina. I congreso internacional de comunicación y género (págs. 951-971). Sevilla: Universidad de Sevilla, Facultad de Comunicación. Consultado https://idus.us.es/bitstream/handle/11441/34591/Pages\%20from\%20LIBR O\%20ACTAS\%20I\%20CONGRESO\%20COMUNICACI\%D3N\%20Y\%20 G\%C9NERO6.pdf;jsessionid=13C19B3F34BCCA20EC7B25812F338CE2? sequence= 1

RAmíREZ, Alejandra. (24 de junio de 2019). Ocurre un suicidio cada 55 horas en Aguascalientes. https://newsweekespanol.com/2019/06/ocurre-un-suicidio-cada-55-horasen-aguascalientes/

Redacción. (11 de mayo de 2015). El municipio de Aguascalientes cuenta con más de tres millones de metros cuadrados de áreas verdes. La Jornada Aguascalientes.

https://www.lja.mx/2015/05/el-municipio-deaguascalientes-cuenta-con-mas-de-tres-millones-de-metros-cuadradosde-areas-verdes/

Redacción. (13 de agosto de 2019). En lo que va de 2019 se han creado 306,014 empleos en Aguascalientes. La Jornada Aguascalientes. https://www.lja.mx/2019/08/en-lo-que-va-de-2019-se-han-creado-306014empleos-en-aguascalientes/

RodRIGUEZ MADERA, Sheilla y Toro, Alfonso José (2002). Ser o no ser: La transgresión del género como objeto de estudio en la Psicología. Avances en psicología clínica latinoamericana (20), 63-78. Consultado en:

https://www.researchgate.net/publication/280054413 Ser o no ser La t ransgresion del genero como objeto de estudio en la Psicologia 
Romo, Alejandro. (17 de julio de 2019). Disminuye percepción de inseguridad en Aguascalientes:

INEGI.

Newsweek.

México.

https://newsweekespanol.com/2019/07/disminuye-percepcion-deinseguridad-en-aguascalientes-inegi/

SolANO, B. L. (2018). Discriminación y racismo en Aguascalientes, ciudad de la gente buena. Ra Ximhai , 14 (2), 149-160. Consultado en: https://www.redalyc.org/jatsRepo/461/46158063009/html/index.html

VALDÉZ, Ricardo. (16 de agosto de 2012). Aguascalientes es el tercer estado en el país con mayor porcentaje de población católica. La Jornada. Aguascalientes. https://www.lja.mx/2012/08/aguascalientes-es-el-tercerestado-en-el-pais-con-mayor-porcentaje-de-poblacion-catolical

\section{Entrevistas}

Entrevista a Bety Vargas el 5 de noviembre de 2019

Entrevista a Crick el 19 de octubre de 2019

Entrevista Domic el 19 de septiembre de 2019

Entrevista a Fatha el 18 de septiembre de 2019

Entrevista a Fer Noriega el 8 de agosto de 2019

Entrevista a Iztheni el 9 de octubre de 2019

Entrevista a Paola Palacios el 11 de octubre de 2019

Entrevista a Sandy de Santos el 8 de octubre de 2019

Entrevista a Venuska el 18 de octubre de 2019

Entrevista a Vanessa Tochin el 10 de octubre de 2019capit. 1 
Ambigua, Revista de Investigaciones sobre Género y Estudios

Culturales, n. ${ }^{\circ}$ 7, 2020, pp. 289-312. ISSN: 2386-8708 